\title{
The Dislocation Mechanism of Stress Corrosion Embrittlement in Ti-6Al-2Sn-4Zr-6Mo
}

\author{
TAMARA P. CHAPMAN, VASSILI A. VORONTSOV, ANANTHI SANKARAN, \\ DAVID RUGG, TREVOR C. LINDLEY, and DAVID DYE
}

\begin{abstract}
An observation of the dislocation mechanisms operating below a naturally initiated hot-salt stress corrosion crack is presented, suggesting how hydrogen may contribute to embrittlement. The observations are consistent with the hydrogen-enhanced localized plasticity mechanism. Dislocation activity has been investigated through post-mortem examination of thin foils prepared by focused ion beam milling, lifted directly from the fracture surface. The results are in agreement with the existing studies, suggesting that hydrogen enhances dislocation motion. It is found that the presence of hydrogen in (solid) solution results in dislocation motion on slip systems that would not normally be expected to be active. A rationale is presented regarding the interplay of dislocation density and the hydrogen diffusion length.
\end{abstract}

DOI: $10.1007 / \mathrm{s} 11661-015-3181-0$

(C) The Minerals, Metals \& Materials Society and ASM International 2015

\section{INTRODUCTION}

TITANIUM alloys are widely used in aerospace applications, as they offer high usable specific fatigue strengths at temperatures up to $\sim 550 \mathrm{deg} .{ }^{[1-4]}$ In the vast majority of cases, titanium alloys are highly corrosion resistant, which has also led to their use in petrochemical and chemical process industries. However, under certain environmental conditions, the adherent and protective $\mathrm{TiO}_{2}$ scale is broken down, exposing the underlying metal to attack. ${ }^{[5]}$ The unwelcome discovery of this problem in the 1960 s led to extensive research, ${ }^{[6-12]}$ which has continued to the present day. ${ }^{[13,14]}$ In 1972, Blackburn et al (in Reference 15) found titanium to be susceptible to stress corrosion cracking (SCC) under a variety of aggressive media, including nitric acid, nitrogen tetroxide, and molten salts. Particular consideration was given to the effect of $\mathrm{NaCl}$ at temperatures above $\sim 300 \mathrm{deg} .{ }^{[9]}$ Titanium was found to be highly vulnerable to SCC in the presence of aqueous sodium chloride, which was of particular concern in aerospace service applications.

Soon after the vulnerability of Ti alloys to SCC was discovered, hydrogen began to be implicated in the

TAMARA P. CHAPMAN, Graduate Student, formerly with Department of Materials, Royal School of Mines, Imperial College London, Prince Consort Road, London SW7 2BP, U.K., is now with Frazer-Nash Consultancy Ltd., Dorking, U.K. VASSILI A. VORONTSOV, Junior Research Fellow, TREVOR C. LINDLEY, Senior Research Investigator, and DAVID DYE, Professor, are with the Department of Materials, Imperial College, South Kensington, London SW7 2AZ, U.K., and also with the Department of Materials, Royal School of Mines, Imperial College London. Contact e-mail: david.dye@imperial.ac.uk ANANTHI SANKARAN, formerly Postdoctoral Research Associate with Department of Materials, Royal School of Mines, Imperial College London, is now with Ford Motor Company Ltd., Brentwood, U.K. DAVID RUGG, Senior Fellow, is with Rolls-Royce plc., Elton Road, Derby DE24 8BJ, U.K.

Manuscript submitted February 14, 2015.

Article published online October 19, 2015 process. Some studies suggested a mechanism involving the production of $\mathrm{HCl}_{(\mathrm{g})}$, and a consequent dissociation of the hydrogen chloride into chloride ions and dissolved hydrogen in solution. ${ }^{[8,9,11]}$ Hydrogen is known be detrimental to the mechanical properties of Ti alloys, including a loss in ductility and enhanced crack growth rates in fatigue. ${ }^{[16]}$ Several mechanisms for this have been offered. Hydrogen has a terminal solubility as high as 50 at. pct in the bec $\beta$-phase, compared to $\sim 7$ at. pct in the hcp $\alpha$-phase. In hydride forming near- $\alpha$ alloys, this might lead to the formation and the cleavage of brittle hydride precipitates. However, the operative mechanism in non-hydride-forming systems is still under debate. Many authors hypothesize that interstitial hydrogen will segregate to $\alpha / \beta$ interfaces, ${ }^{[17]}$ where it might weaken the cohesive strength (hydrogen-enhanced decohesion, HEDE), resulting in intergranular separation of the interfaces. ${ }^{[10,18,19]}$ Shih et al. ${ }^{[20]}$ consider there to be minimal experimental evidence supporting this interface decohesion theory. Instead, they propose an alternative mechanism, supporting previous work by Beachem, where hydrogen-enhanced localized plasticity (HELP) in the vicinity of the crack tip is suggested. ${ }^{[21,22]}$ Another postulated mechanism is adsorption-induced dislocation emission (AIDE), where hydrogen adsorbed within a few atomic distances of the crack tip results in dislocation emission from the crack tip. ${ }^{[23-25]}$ The difference between the AIDE and the HELP mechanisms chiefly concerns whether hydrogen diffusion into the bulk is implied.

Not only is hydrogen more soluble in the beta phase, it is also able to diffuse much more rapidly in the body-centered cubic (bcc) $\beta$ structure than in the close-packed $\alpha$ phase. ${ }^{[26]}$ In two-phase $\alpha / \beta$ titanium alloys, a continuous beta phase is then supposed to provide a "short circuit path" for hydrogen transport. ${ }^{[18,19,27]} \mathrm{Pao}$ and O'Neal ${ }^{[28]}$ suggested that hydrogen diffusion in the continuous beta ligaments of Ti-6242S 
could be the rate-controlling process for hydrogen-enhanced fatigue crack growth. Thus, lamellar microstructures with continuous $\beta$-phase could be more vulnerable to hydrogen effects.

The $\alpha / \beta$ alloy, Ti-6Al-2Sn-4Zr-6Mo (Ti-6246-wt pct), offers an increase in the operating temperature of up to $150 \mathrm{~K}$ compared with the workhorse alloy, Ti-6Al-4V (Ti-64-wt pct), predominantly due to its high strength owing to the formation of fine-scale secondary $\alpha$. Therefore, Ti-6246 is now commonly used in applications up to $500 \mathrm{~K}$, whereas Ti-64 is restricted to cooler locations in the jet engine.

In a companion study, ${ }^{[29]}$ we examined the formation mechanism of a blue-colored fatigue crack initiation feature observed at the origin of several fracture surfaces from elevated temperature spinning rig and test specimens, some of which were found to have reduced fatigue lives. Evidence of chlorine within the "blue spot" was observed by scanning transmission electron microscopy-based energy dispersive X-ray analysis (STEM-EDX). The blue spot was also found to possess a thick $(\sim 200 \mathrm{~nm}) \mathrm{TiO}_{2}$ oxide scale. Further evidence for ${ }^{35} \mathrm{Cl}^{-},{ }^{23} \mathrm{Na}^{+}$, and ${ }^{16} \mathrm{O}^{-}$was found using focused ion beam-secondary ion mass spectrometry (FIB-SIMS). A hot-salt stress corrosion cracking (HSSCC) mechanism was proposed as an explanation for the apparent change in the fracture mode seen at the fatigue crack origin.

In summary, the corrosion mechanism was held to operate as follows. First, the $(\mathrm{NaCl})$ salt deposit reacts with atmospheric moisture and the oxide to produce $\mathrm{HCl}_{(\mathrm{g})}$ and sodium titanate. This reaction disrupts the oxide scale, allowing the $\mathrm{HCl}$ to then react with the exposed metal, forming titanium alloy chlorides and hydrogen. The volatile alloy chlorides can then escape from the corrosion crack to react with moisture, precipitating a $\mathrm{TiO}_{2}$ corrosion product and re-forming $\mathrm{HCl}$. The hydrogen produced can then diffuse into the metal at the crack tip, where it remains because hydrogen concentrates at regions of high stress triaxiality. ${ }^{[12,30]}$ Therefore, the extent of embrittlement is controlled by a competing reaction of hydrogen charging vs hydrogen diffusion, with hydrogen assisting the cracking process. Eventually, and with a finite supply of salt, the $\mathrm{Cl}$ supply will become exhausted due to losses from the system, resulting in a decrease in hydrogen charging. Therefore, beyond a certain crack length, a transition from HSSCC to conventional fatigue crack propagation was observed.

Therefore, it remains to explain how the hydrogen embrittling mechanism operated in practice, through HELP or otherwise, in this naturally initiated cracking process. Previously, Robertson and coworkers have used hydrogen-containing in situ straining cells in the TEM to examine the effect of hydrogen on dislocation activity. ${ }^{[20,31-33]}$ These studies suggested that hydrogen enhances dislocation motion by locally reducing the stress required to cause motion and/or increasing the velocity. Shih et al. showed that by adding and removing hydrogen from the environment cell, they could control slip activity at the crack tip. ${ }^{[20]}$ Hydrogen shielding was proposed as a rationale for this enhanced mobility. ${ }^{[31-33]}$

In the current study, we examine the dislocations underlying the "blue spot" corrosion fatigue crack origin, compared with the low-cycle fatigue situation, to determine if a change in dislocation mechanisms is observable. This will be of particular utility for the investigation of failures where hydrogen-enhanced cracking is suspected to be involved.

\section{MATERIAL AND EXPERIMENTAL PROCEDURES}

The Ti-6246 material used during this study was provided by Rolls-Royce plc., and was tested at the Mechanical Testing Operations Center (MTOC), Rolls-Royce Deutschland. Two specimens were examined ("blue spot" specimen U7-086 $6^{[29]}$ and "datum" specimen LCF127 ) which were tested at 450 deg with a maximum stress $\left(\sigma_{\max }\right)$ of $600 \mathrm{MPa}$ (U7-086) and $640 \mathrm{MPa}$ (LCF127) and an $\mathrm{R}$ ratio of 0.1 . The material was processed with a final heat treatment in the $\beta$ phase field followed by aging in the $\alpha+\beta$ phase field, to produce the lamellar microstructure shown in Figure 1. The microstructure consisted of coarse, high aspect ratio primary $\alpha$ laths, and fine secondary $\alpha$ laths residing within a retained $\beta$ matrix. Fully lamellar microstructures are used to maximize fracture toughness, ${ }^{[5]}$ with elevated strength provided by the fine secondary alpha phase.

The spatial and crystallographic orientations of facetted fracture features at the hydrogen-embrittled origin were determined using quantitative tilt fractography (QTF) and electron backscattered diffraction (EBSD). A Zeiss Auriga field emission gun-secondary electron microscope (FEG-SEM), fitted with an Oxford Instruments EBSD detector, was used. By identifying the spatial orientation of the fracture features using QTF, ${ }^{[34-36]}$ it was possible to index Kikuchi diffraction patterns obtained directly from the fracture surface. Two facets within the hydrogen-embrittled initiation site were analyzed, Figure 2.

In situ FIB lift out was used for the preparation of thin foils from the "blue spot" origin in U7-086, Figure 2, from the crack initiation site in LCCF127 and from the region of fatigue crack growth in U7-086, $300 \mu \mathrm{m}$ from the origin. FIB milling was carefully performed so as to minimize the surface damage due to irradiation by the $\mathrm{Ga}^{+}$beam, ${ }^{[37,38]}$ commonly attributed to $\mathrm{Ga}^{+}$ion implantation, which can, in some cases, affect defect structures. Initially, a sample was produced from the as-received material that had not undergone further deformation since forging, using a dual beam FEI Helios NanoLab 600. Approximately $2 \mu \mathrm{m}$ of platinum was deposited onto the region of interest to protect the surface from the $30 \mathrm{kV}$ primary $\mathrm{Ga}^{+}$beam. After lift-out, the sample was thinned down to $\sim 180 \mathrm{~nm}$. The final thinning stage involved low current ( $28 \mathrm{pA}$ ), low energy ( $2 \mathrm{keV}$ ) polishing, to remove $\sim 20 \mathrm{~nm}$ of material from the front and back of the sample. A comparison sample was prepared from the same as-received material using conventional electropolishing with a solution of 3 pet perchloric acid and 40 pet butan-1-ol in 57 pet methanol at $24 \mathrm{~V}$ and $-40{ }^{\circ} \mathrm{C}$. Both samples 

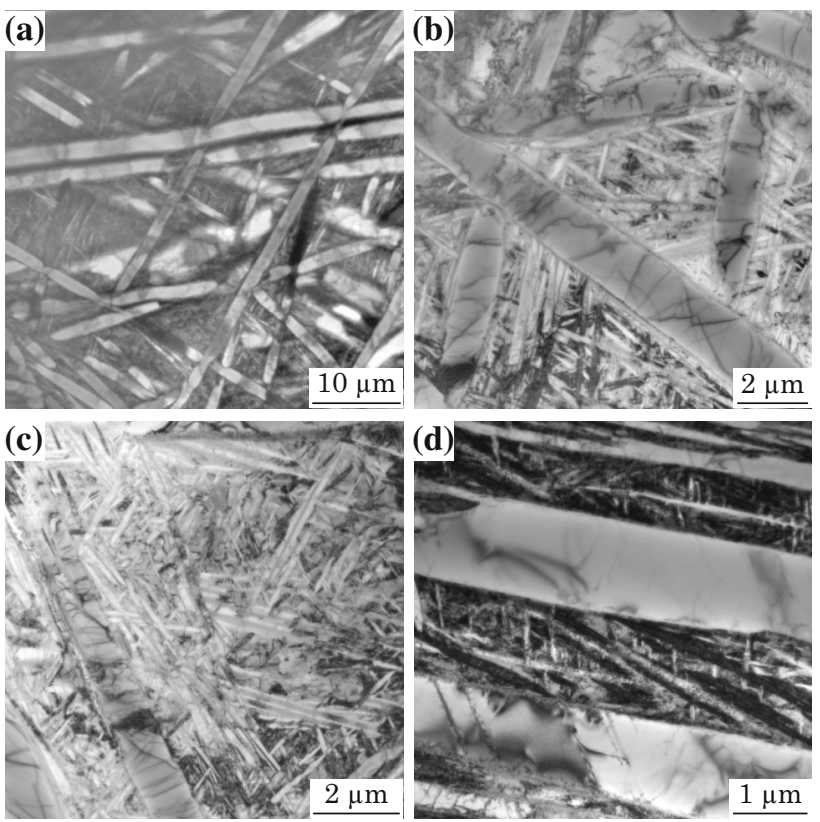

Fig. 1-Bright field (BF) TEM images: (a) Overview of lamellar $\alpha / \beta$ microstructure, $(b)$ and $(c)$ Primary and secondary alpha laths, $(d)$ Secondary alpha residing between coarse primary alpha laths.

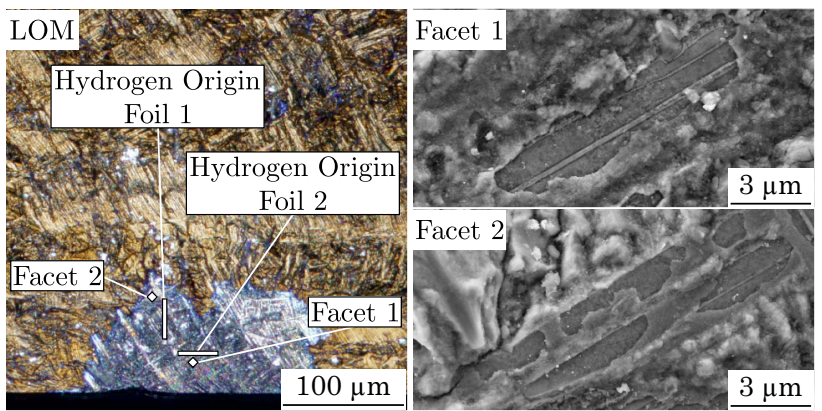

Fig. 2-The hydrogen-embrittled zone is demonstrated by the blue semi-elliptical feature at the fatigue crack origin. The locations of the facets for the QTF/EBSD technique, as well as the position of the foils for dislocation contrast analysis, are given.

provided consistent results in the TEM, see Section IV-A and Figure 10. All site-specific samples were then lifted directly from the fracture surface using the same FIB lift-out procedure. The optimal sample thickness was found to be $\sim 180 \mathrm{~nm}$. This was thick enough to observe clear Kikuchi lines, while achieving adequate imaging conditions. For consistency, all analyses were undertaken on primary alpha laths, as shown in Figure 3.

Dislocations within grains connected to the fracture surface were examined using a combination of invisibility contrast conditions, alongside trace analysis, using a $200 \mathrm{keV}$ JEOL 2000FX TEM. The grains of interest were imaged with $g=\{0002\},\{0 \overline{1} 11\}$ and $\{0 \overline{1} 1 \overline{1}\}$, as well as $\{10 \overline{1} 0\},\{\overline{2} 110\}$ and $\{0 \overline{1} 12\}$ if possible, where $g$ is the reciprocal lattice vector. The Burgers vectors, $b$, of screw dislocations were then differentiated by calculating invisibility contrast values as the scalar product of the reciprocal lattice vector and possible Burgers vectors
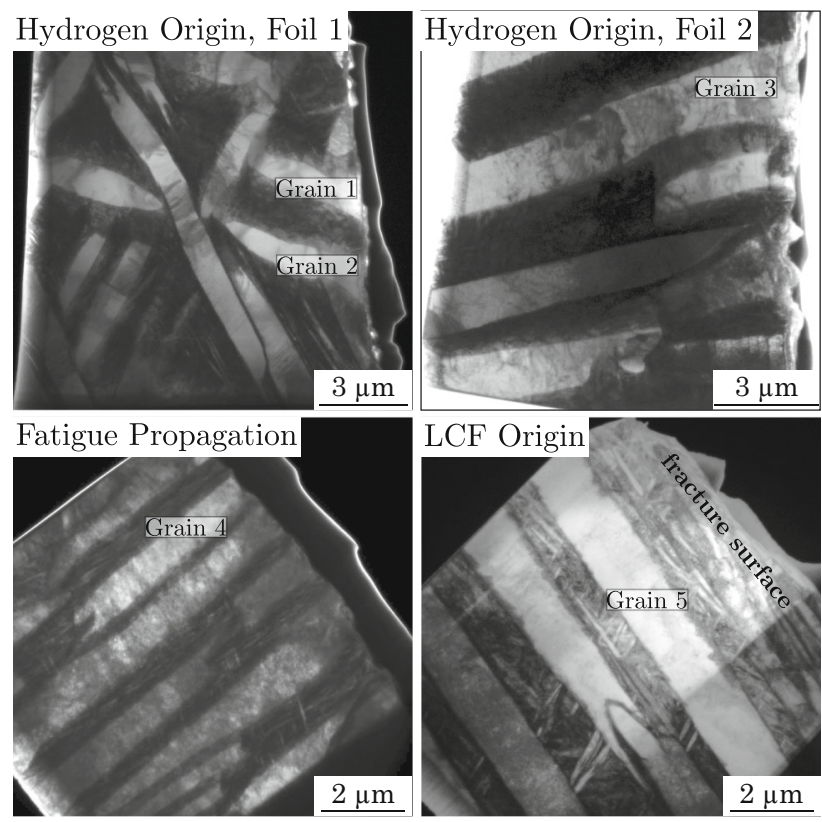

Fig. 3-Position of the grains of interest within the respective FIB-prepared TEM foils.

$(g \cdot b)$. Edge dislocations were analyzed by considering contrast values calculated by $g \cdot(b \times l)$, where $l$ is the line vector. By incorporating trace analysis, it was possible (in some cases) to determine the active slip system. Considering the orientation of the grain with respect to the fatigue loading axis, Table I, comparisons with favorable slip systems according to Schmid factor calculations could also be made.

\section{RESULTS}

\section{A. Facet Orientation}

The spatial and crystallographic orientations of two facets residing within the hydrogen-embrittled initiation site were analyzed using QTF/EBSD. It was found that facet 1 and facet 2 were spatially orientated such that each facet normal was $<10$ deg away from the tensile loading axis. By accounting for the angular deviation of the facet normal away from the loading direction in the EBSD, the crystallographic orientation of each facet could then be acquired. This made it possible to index directly from the fracture surface without the need for prior sectioning and surface preparation. With reference to the [0001] pole figures, demonstrated in Figure 4, it can be seen that both facet normals lie close to the [0001] direction. In fact, the facet normals are measured to be orientated $\sim 14$ deg away from the [0001] direction, indicating that the plane of the facet, and thus fracture plane, coincides with either the $\{10 \overline{1} 7\}$ or $\{10 \overline{1} 8\}$ planes, inclined at 15 and 13 deg away from the basal plane, respectively. Both the $\{10 \overline{1} 7\}$ and $\{10 \overline{1} 8\}$ planes are known to be common hydrogen-habited planes, ${ }^{[39]}$ indicating that the fracture mechanism is likely to have been modified by the presence of hydrogen. It should be 
Table I. Location and Orientation of Grains, where $\theta$ is Defined as the Angle Between the Loading Direction and the c-Axis

\begin{tabular}{llc}
\hline Grain ID & \multicolumn{1}{c}{ Description } & $\theta(\mathrm{deg})$ \\
\hline 1 & Hydrogen-embrittled LCF Origin & 71 \\
2 & Hydrogen-embrittled LCF Origin & 71 \\
3 & Hydrogen-embrittled LCF Origin & 25 \\
4 & LCF Crack Propagation & 31 \\
5 & LCF Origin & 77 \\
\hline
\end{tabular}

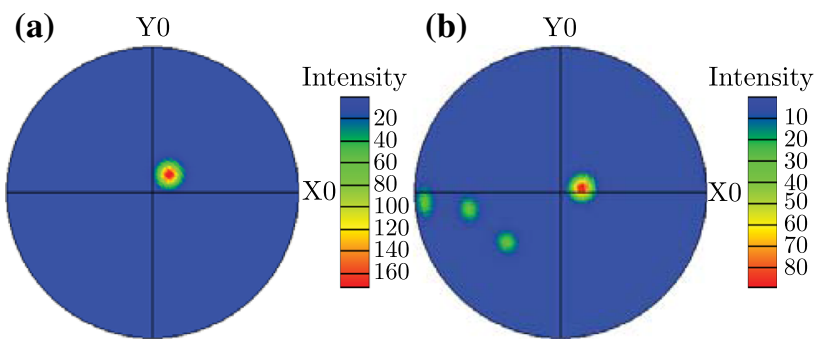

Fig. 4-[0001] pole figures obtained from facets within the hydrogen-embrittled origin: (a) Facet $1 \sim 14$ deg from the basal plane, $(b)$ Facet $2 \sim 14$ deg from the basal plane.

noted that the three low-intensity spots in Figure 4(b) have arisen from indexing of an adjacent, and differently orientated grain.

\section{B. Dislocation Contrast Analysis}

Three FIB-prepared foils, containing grains 1 to 4 , were lifted directly from the low-cycle fatigue fracture surface that suffered HSSCC (sample U7-086). Grains 1, 2 and 3 are associated with an area embrittled by hydrogen, near the fatigue crack origin; Figure 2 (LOM) demonstrates the location of the foils within the hydrogen-embrittled origin. Grain 4 resided within a foil that was milled from an area of fatigue crack propagation, approximately $600 \mu \mathrm{m}$ from the origin. Finally, grain 5 was observed within a foil that was milled from the origin of a different LCF specimen that had not undergone hydrogen embrittlement (sample LCF 127). To have the best chance of observing dislocation mechanisms associated with the fracture process, it was deemed important to analyze grains connected to the fracture surface, i.e. not subsurface; Figure 3 shows the positions of the analyzed grains with respect to the fracture surface.

\section{Hydrogen-assisted fatigue cracking}

Grain 1s $\{0002\}$ plane normal was found to be $\sim 70$ deg to the tensile-fatigue loading axis. Figure 5(a) shows bright field (BF) TEM micrographs, imaged with several diffraction conditions. For $g_{0002}$, an array of linear dislocation segments were observed across the width of the primary alpha grain, ranging from $\sim 700 \mathrm{~nm}$ to $1.5 \mu \mathrm{m}$ beneath the fracture surface. The dislocations appear to be orientated almost parallel to the (0002) trace, obtained from the $\langle 1 \overline{2} 10\rangle$ selected area diffraction pattern (SADP). Referring to the 4-index hcp coordinate

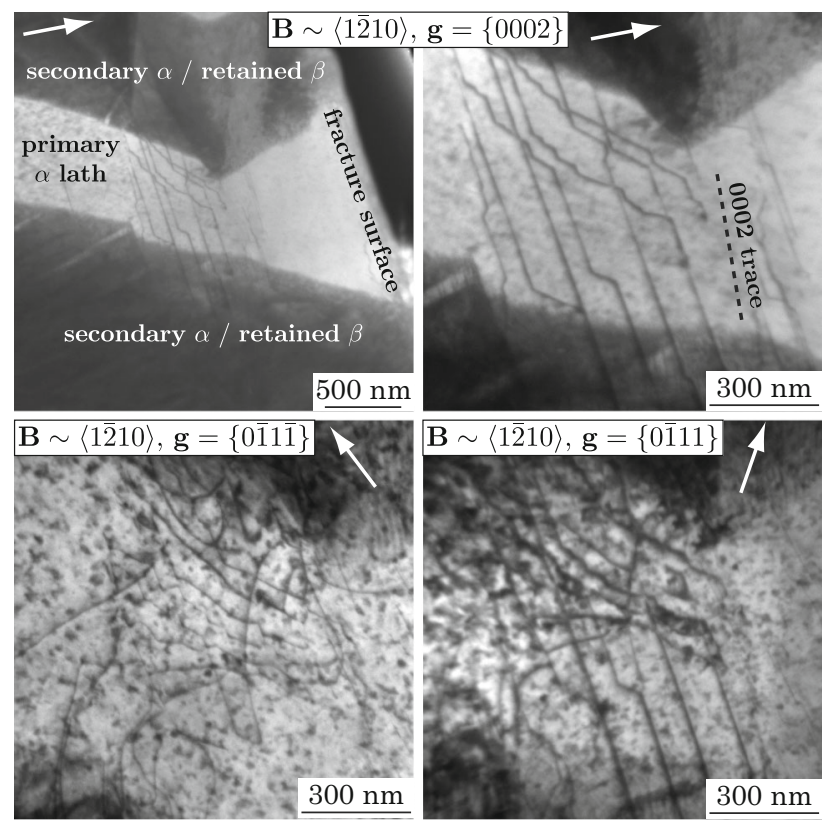

Fig. 5-BF dislocation contrast images for grain 1, for diffraction conditions of $g_{0002}, g_{0 \overline{1} 1 \overline{1}}$ and $g_{0 \overline{1} 11}$. The white arrows indicate the direction of the $g$ vector.

system, it can be said that the beam direction vector $(B)$ lies in the basal plane of the crystal and is parallel to two of the $\langle a\rangle$ type Burgers vectors. Any dislocations residing in the basal plane, and imaged under these conditions edge-on, would appear as straight line segments, parallel to the (0002) trace. Slight misorientation between the trace and the dislocation segment is likely to result from the small rotation of the SADP (from which the trace is obtained) when the sample is tilted away from the pole to reach the required $g$ vector. From trace analysis, it is therefore implied that the array of dislocations in Figure $5\left(g_{0002}\right)$ has resulted from slip on the basal plane.

Basal glide has frequently been observed in $\alpha$ titanium, but this occurs almost exclusively with $\langle 11 \overline{2} 0\rangle$ type Burgers vectors. Under $g_{0002}$ diffraction conditions, as in Figure 5 (upper), all screw dislocations with $\langle a\rangle$ type $\langle 11 \overline{2} 0\rangle$ Burgers vectors should have zero contrast, and thus be invisible, which is not the case here. It then became necessary to evaluate the edge component also, incorporating the $g \cdot(b \times l)$ criterion. Introducing a perpendicular line vector, with indices $\langle 1 \overline{1} 00\rangle$, demonstrates positive contrast for edge dislocations gliding on (0002) planes, when imaged with $g_{0002}$. However, for all the $\{1 \overline{1} 00\}$ glide planes, there is zero contrast for dislocations with $\langle 11 \overline{2} 0\rangle$ Burgers vectors. This finding eliminates the possibility of prismatic slip in this case. Hence, considering the contrast criteria and the minimal misorientation between the (0002) trace and the dislocation segments, one possibility is that the linear dislocations segments in Figure $5\left(g_{0002}\right)$ are associated with $\langle 11 \overline{2} 0\rangle\{0001\}$ type slip systems, and have edge character.

An alternative hypothesis could be that these dislocations are of $\langle c+a\rangle$ type. Both edge and screw 
character $\langle c+a\rangle$ dislocations have contrast at imaging conditions of $g_{0002}$. Glide in the direction of such Burgers vectors is restricted to the pyramidal planes $\{10 \overline{1} 0\}$ and $\{11 \overline{2} 2\}$. Under imaging conditions of $B \sim\langle 2 \overline{1} 10\rangle$, these inclined planes would be viewed at a small angle to the face of the plane, rather than edge on, and hence may not be expected to appear as straight lines as they do for conditions of $g_{0002}$. However, $\langle c+a\rangle$ dislocations have an inherently higher elastic energy compared with the $\langle a\rangle$ type. Hence, it is reasonable to expect that such dislocations could exist as straight lines to minimize this energy, rather than the bowed out segments and loops that may be observed from $\langle a\rangle$ type line defects.

The same area was viewed at alternative diffraction conditions of $g_{0 \overline{1} 1 \overline{1}}$ and $g_{0 \overline{1} 11}$ in an attempt to determine the active dislocation type. Contrast of the original linear dislocation array, as seen for $g_{0002}$, remains for both $g_{0 \overline{1} 1 \overline{1}}$ and $g_{0 \overline{1} 11}$, in varying intensities. This observation essentially excludes edge $\langle c+a\rangle$ dislocations gliding on first-order pyramidal planes, since the invisibility criterion suggests zero contrast for either $g_{0 \overline{1} 1 \overline{1}}$ or $g_{0111}$, in most cases. Table II provides details of the invisibility criterion for a number of $g$ vectors. Furthermore, the elastic energy of a dislocation is proportional to the magnitude of the Burgers vector, with the $\langle c+a\rangle$ energy almost twice that of the $\langle a\rangle$-type Burgers vector. Finally, edge $\langle a\rangle$ shows zero contrast at $g_{0002}$ for prismatic planes and at $g_{01111}$ for first-order pyramidal planes. Hence, it is tentatively suggested that the linear dislocation arrays observed for grain 1 in Figure 5 are associated with edge dislocations undergoing basal slip in the direction of $\langle 11 \overline{2} 0\rangle$ type Burgers vectors.

Closer investigation of Figure 5(b) reveals a number of shorter dislocation segments orientated $\sim 120$ deg to the (0002) trace. Both the first and second-order pyramidal slip planes are inclined $\sim 120$ deg to the basal plane. First-order $\{1 \overline{1} 01\}$ planes are able to slip along either $\langle c+a\rangle$ type $\langle 11 \overline{2} 3\rangle$ or $\langle a\rangle$ type $\langle 11 \overline{2} 0\rangle$ Burgers vectors, whereas second-order $\{\overline{1} 122\}$ planes are restricted to glide in the $\langle c+a\rangle$ direction only. Under certain diffraction conditions, the short angled segments move out of contrast while those longer sections, parallel to the (0002) trace, remain in contrast. It is hence implied that the short, orientated segments observed have a different Burgers vector to the remainder of the linear array.
The observations made from analysis of grain 2 are consistent with those from grain 1, which has the same crystal orientation. The uppermost image in Figure 6 shows the full length of the primary alpha lath, identified as grain 2 . In this case, the straight dislocation segments are observed up to $3 \mu \mathrm{m}$ beneath the fracture surface. Beyond this depth, similar dislocations are not observed. In fact, beyond $3 \mu \mathrm{m}$ below the surface, only a small group of differently orientated dislocations can be identified at the very tip of the primary alpha lath. Presumably, these have arisen as a result of grain 2 growth during processing, and the consequent collision with another primary alpha lath; refer to Figure 3, hydrogen origin foil 1 . These observations again suggest that these dislocation structures are restricted to material only a few microns subsurface of the growing crack. Observations based on trace analysis and invisibility contrast suggest that, like grain 1 , the linear dislocation segments spanning across the lath have edge character, and have arisen from $\langle 11 \overline{2} 0\rangle\{0001\}$ type slip. Imaging under $g_{011 \overline{1}}$ repeats these findings; Figure 6. With

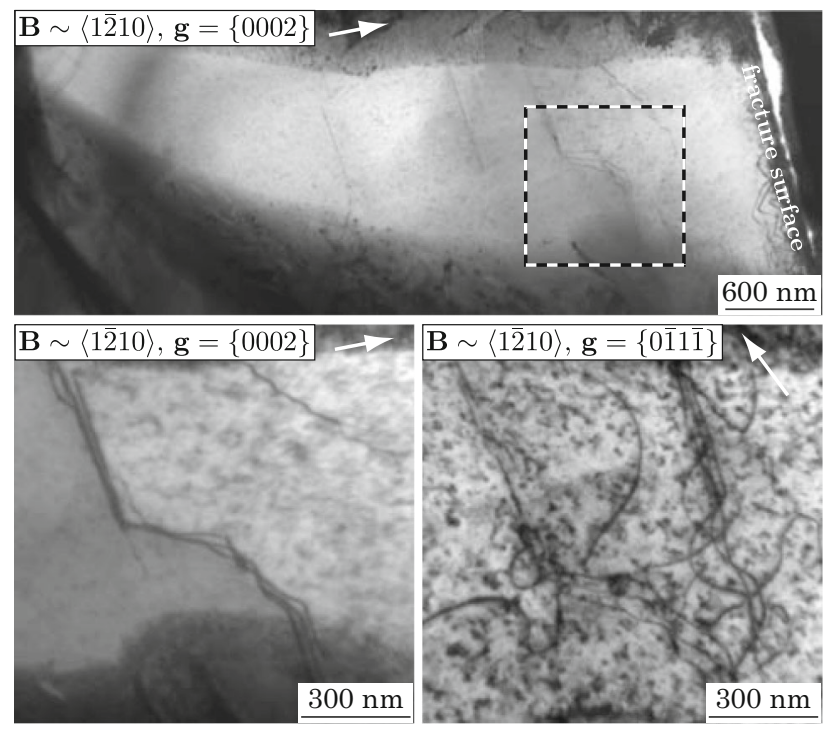

Fig. 6-BF dislocation contrast images for grain 2: (upper) Stitch of low-magnification images for $g_{0002}$; (lower) Increased magnification views for $g_{0002}$ and $g_{0 \overline{1}} \overline{1}$. The dashed box indicates the area magnified from the stitch in the lower left image. In all cases, the white arrow indicates the direction of the $g$ vector.

Table II. Demonstrating Selected Invisibility Contrast Values, where Contrast is Defined as + and Invisibility is Defined as 0

\begin{tabular}{|c|c|c|c|c|c|c|c|c|c|}
\hline \multirow[b]{2}{*}{ Character } & \multirow[b]{2}{*}{ Type } & \multirow[b]{2}{*}{$b$} & \multirow[b]{2}{*}{ Glide Plane } & \multicolumn{6}{|c|}{ Invisibility Contrast } \\
\hline & & & & $g_{0002}$ & $g_{0 \overline{1} 1 \overline{1}}$ & $g_{0 \overline{1} 11}$ & $g_{0 \overline{1} 12}$ & $g_{\overline{2}}{ }_{110}$ & $g_{10 \overline{1} 0}$ \\
\hline Screw & $\langle a\rangle$ & $\frac{1}{3}[\overline{1} \overline{1} 20]$ & $(0001)$ or $(10 \overline{1} 0)$ or $(\overline{1} \overline{1} 01)$ & 0 & + & + & + & + & + \\
\hline Screw & $\langle c+a\rangle$ & $\frac{1}{3}[1 \overline{2} 13]$ & $(\overline{1} 122)$ or $(\overline{1} 011)$ & + & + & + & + & + & 0 \\
\hline Edge & $\langle a\rangle$ & $\frac{1}{3}[\overline{1} 120]$ & $(0001)$ & + & + & + & + & 0 & 0 \\
\hline Edge & $\langle a\rangle$ & $\frac{1}{3}[\overline{1} \overline{1} 20]$ & $(\overline{1} 100)$ & 0 & + & + & + & + & + \\
\hline Edge & $\langle a\rangle$ & $\frac{1}{3}[\overline{1} \overline{1} 20]$ & $(\overline{1} 101)$ & + & + & 0 & + & + & + \\
\hline Edge & $\langle c+a\rangle$ & $\frac{1}{3}[1 \overline{2} 13]$ & $(\overline{1} 101)$ & + & + & 0 & + & + & + \\
\hline
\end{tabular}


reference to the high magnification micrograph for $g_{0002}$ given in Figure 6, the short, inclined dislocation segments first observed in grain 1 , are indicated again in grain 2. In this instance, at least three individual linear dislocations can be resolved, with each inclining away from the (0002) trace at angles between $\sim 105$ to $120 \mathrm{deg}$. A collection of nonlinear dislocations can also be identified directly beneath the fracture surface of grain 2 for conditions of $g_{0002}$; see low magnification image. These looping dislocations appear to have been emitted from the 200-nm-thick oxide layer that covers the hydrogen-embrittled origin. It is, however, unclear whether these dislocations were present prior to thin foil preparation, and may in fact be a consequence of the platinum deposition process. For this reason they will not be discussed in more detail.

The $c$-axis of grain 3 is inclined at $\sim 25 \mathrm{deg}$ to the fatigue loading axis, compared to a 70-deg inclination for both grains 1 and 2. The contrast observed for grain 3 at each diffraction condition can be described by the invisibility criterion given for edge $\langle a\rangle \frac{1}{3}[\overline{1} 120]$ and edge $\langle c+a\rangle \frac{1}{3}[1 \overline{2} 13]$ dislocations, gliding on (1101) planes in both cases; as given in Table II. Since dislocation contrast is observed for $g_{0002}$ and $g_{10 \overline{1} 0}$, both screw $\langle a\rangle$ and $\langle c+a\rangle$ dislocations can be excluded. Similarly, strong contrast for $g_{\overline{2} 110}$ and $g_{0002}$ eliminates edge $\langle a\rangle \frac{1}{3}[\overline{1} 120]$ dislocations gliding on basal and prismatic planes, respectively; refer to Figure 7 . It is therefore suggested that dislocations are moving on the first-order pyramidal (1101) plane. Considering the increased elastic energy of $\langle c+a\rangle$ dislocations compared with the $\langle a\rangle$, it is not unreasonable to expect the dislocations to be $\langle a\rangle$ type in nature.

Figure 7 presents increased magnification micrographs of a bright field image for $g_{0 \overline{1} 12}$. Two rows of

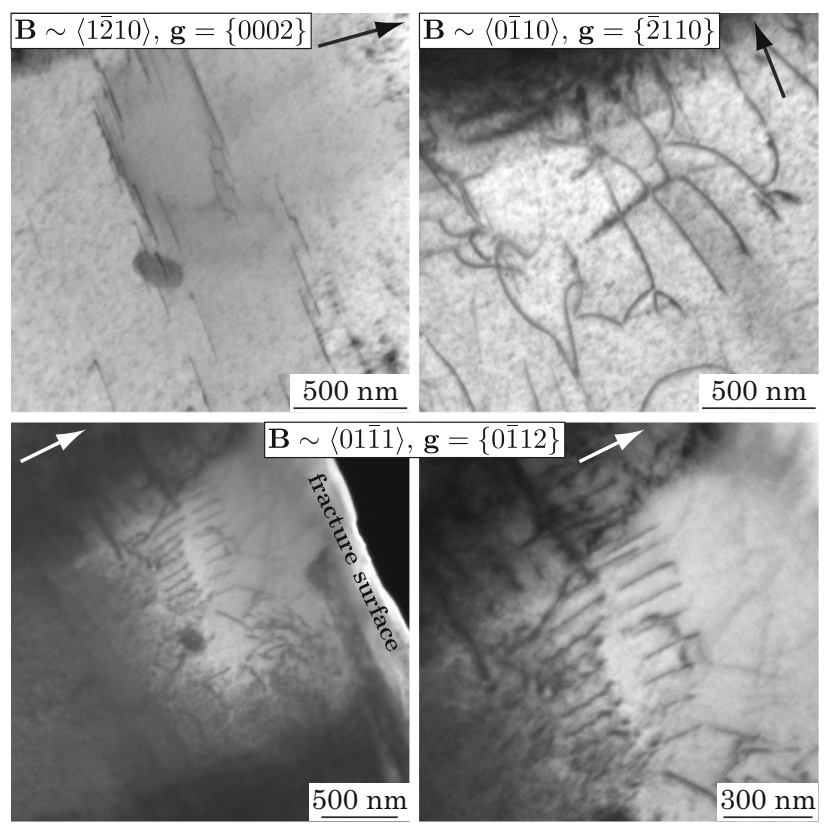

Fig. 7-BF dislocation contrast images for grain 3, for diffraction conditions of $g_{0002}, g_{\overline{2} 110}$ and $g_{0112}$. The arrows indicate the direction of the $g$ vector. parallel dislocation arrays are seen to give contrast at these imaging conditions, as discussed above. Consistent with findings from grains 1 and 2, the dislocations only reside immediately beneath the fracture surface, up to $\sim 15 \mu \mathrm{m}$ subsurface. Considering the FIB foil in $3 \mathrm{D}$, but noting its transparency, it is likely that the discontinuous appearance of the dislocation segments is an effect of the position and orientation of the dislocations within the foil. Moreover, because the dislocations do not lie in the plane of the foil, and thus are restricted to the foil thickness, they appear in short segments where they "run out" the front and back planes of the foil. The two rows observed here, are considered to be dislocations residing on parallel and adjacent sets of planes.

\section{Fatigue crack propagation}

Grain 4 was located within a thin foil that was lifted from the U7-086 sample fracture surface at a crack length of $\sim 600 \mu \mathrm{m}$. As the crack exceeded $\sim 100 \mu \mathrm{m}$ in length, there was an observed transition in the cracking mechanism from HSSCC near the origin, to low-cycle fatigue crack propagation. Grain 4 has therefore not experienced any effect of solute hydrogen. Since grains 3 and 4 have similar crystallographic orientations $\{0002\}$ normal $\sim 25 v s \sim 30$ deg from the loading axis), direct comparisons can be made between them. Figure 8 provides BF diffraction contrast images taken with diffraction conditions of $g_{0002}$. A high dislocation density is seen to extend down the length of the primary alpha laths. This is in stark contrast to observations from hydrogen-embrittled grains. For grains 1-3, a low density of individual dislocation arrays were observed, and appeared to be restricted to the first few microns, directly beneath the fracture surface. The low magnification image given in Figure 8 has been included to demonstrate these differences. Although not included here, images showing similarly high dislocation densities were also obtained for several other diffraction conditions. A high dislocation density visible for several $g$ vectors indicates that a large number of dislocations were active during the deformation process. As the dislocations move they will interact with each other, and likely entangle as they intersect.

Unlike the hydrogen-embrittled grains 1 to 3, where a single slip system could be identified, it is likely that dislocations with different Burgers vectors and gliding on various planes, has led to the high densities observed

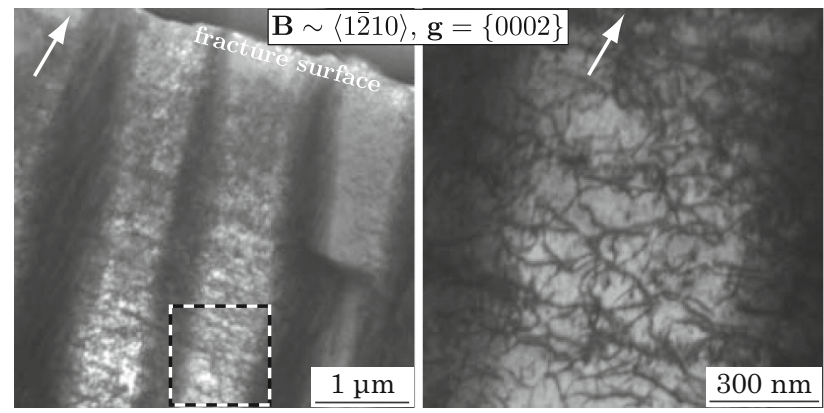

Fig. 8-BF dislocation contrast images for grain 4, for diffraction conditions of $g_{0002}$. The white arrows indicate the direction of the $g$ vector. 
in grain 4 and the adjacent laths. However, detailed examination of Figure 8 reveals that the bands of the most highly dense dislocation entanglements appear to be almost parallel to the (0002) trace. By observing the highest density bands in neighboring primary alpha grains, it is suggested that these dislocations may have been transmitted across the secondary alpha/beta matrix ligaments, and continue into the adjacent primary alpha lath.

\section{Comparator low-cycle fatigue origin}

Figure 9 illustrates the dislocations observed in a primary $\alpha$ lath (grain 5) beneath the origin of a low-cycle fatigue test specimen not subjected to HSSCC, in similar testing conditions. A low magnification view of the TEM foil is provided in Figure 3. A high density of dislocations can be observed for $g_{0 \overline{1} 11}$, in common with the low-cycle fatigue propagation region (grain 4) already examined. The c-axis of grain 5 is inclined at $\sim 80 \mathrm{deg}$ to the fatigue loading axis, and therefore, grain 5 can be directly compared with grains 1 and 2; see Table I. The observation of a higher dislocation density in the low-cycle fatigue crack growth region is consistent with those observed in a conventional low-cycle fatigue origin, and quite distinct from those in the hot-salt stress corrosion crack origin region. Thus, an effect of crack length can be excluded as an explanation for the observations made.

\section{DISCUSSION}

\section{A. Comparability of FIB and Electropolished TEM Foils}

Several studies have discussed the surface damage associated with FIB-prepared thin foils. When the gallium beam impinges on the sample surface, $\mathrm{Ga}^{+}$ ions are implanted in the lattice of the target material. This can lead to local compositional variations, as well as amorphization of the outer layers of the foil. ${ }^{[37]}$ Gallium penetration depth increases with beam energy (accelerating voltage). More $\mathrm{Ga}^{+}$interstitials would therefore be expected after bombardment at $30 \mathrm{keV}$ compared with $5 \mathrm{keV}$. These interstitial $\mathrm{Ga}^{+}$ions impose strain on the crystal lattice and hence generate strain contrast in the TEM. Kiener et al. ${ }^{[38]}$ reported

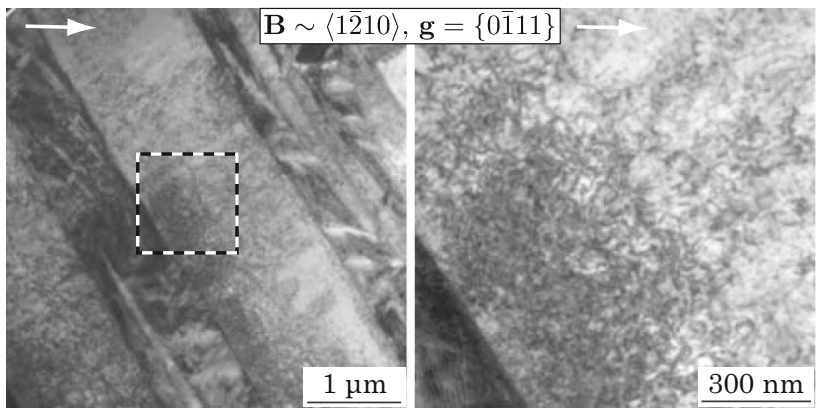

Fig. 9-BF dislocation contrast images for grain 5, for diffraction conditions of $g_{0111}$. The white arrows indicate the direction of the $g$ vector. that after milling at $30 \mathrm{keV}$, it was not possible to distinguish individual preexisting dislocations from the contrast associated with point defects, in the form of $\mathrm{Ga}^{+}$interstitials. However, FIB-induced damage can be controlled by reducing the beam energy. Although Kiener et al. still observed some surface damage after irradiation at $5 \mathrm{keV}$, all preexisting defects remained visible. In the present study, final milling at $28 \mathrm{pA}$ and $2 \mathrm{keV}$ was undertaken in an attempt to remove damaged outer layers, while minimizing further damage.

Figure 10 demonstrates two different imaging conditions for: (1) a primary alpha lath within a FIB-prepared foil, and (2) a primary alpha lath within an electropolished foil. Both samples were prepared from the as-received material that had undergone no further mechanical deformation since the forging process. For invisibility conditions of $g_{0002}$, both samples show a low density of linear dislocation segments, which extend across the width of grain. For both preparation techniques, the dislocations are also distributed throughout the length of the primary alpha. For an alternative contrast condition of $g_{0 \overline{1} 11}$, the dislocation density and distribution is again comparable for the grains in either sample, as is the general appearance of the dislocation segments. Evidence of FIB damage can be seen at both imaging conditions, but it is rather more severe for $g_{0 \overline{1} 11}$. The supposed FIB-induced damage can be observed as the speckled contrast throughout the alpha lath, which is likely to be associated with strain accumulation caused by $\mathrm{Ga}^{+}$ions implanted during milling. As a result, the dislocations within the FIB foil are slightly less distinctive than those in the electropolished foil. However, the dislocation contrast in the FIB foil remains adequate, so it is possible to differentiate

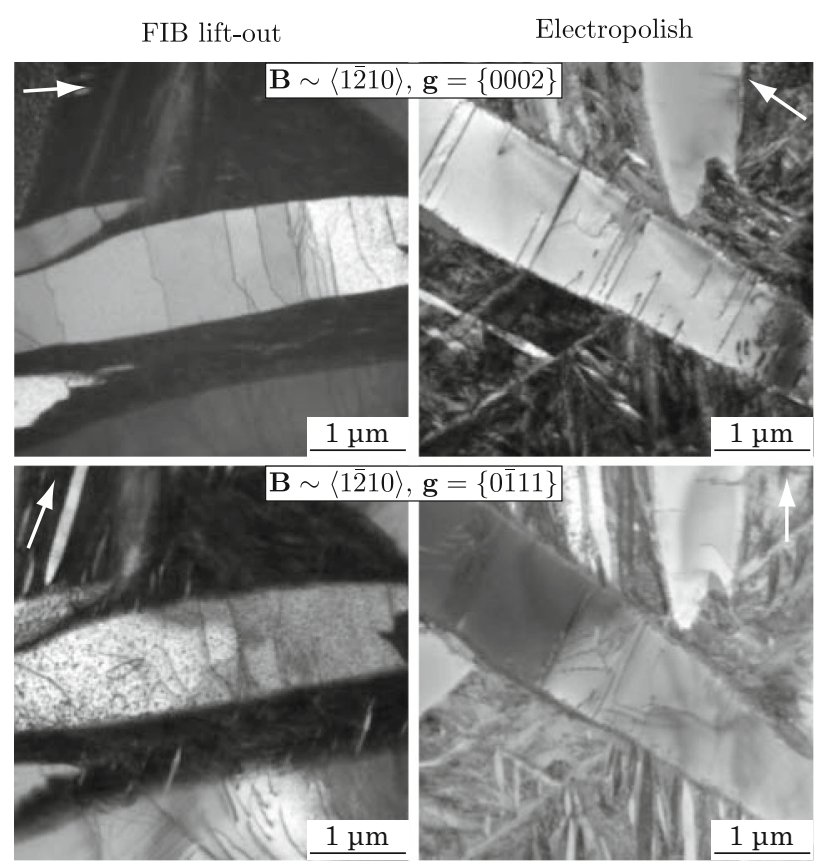

Fig. 10-BF dislocation contrast images for FIB and electropolished prepared thin foils, for diffraction conditions of $g_{0002}$ and $g_{0111}$. The white arrows indicate the direction of the $g$ vector. 
between the dislocations and the FIB-induced damage. It is hence concluded that preparation using FIB milling has not affected the ability to analyze dislocations within $\alpha$ titanium laths.

\section{B. Facet Formation}

Over the past few years, various research groups have raised questions concerning the validity of the common association of planar facet features with a brittle, cleavage, fracture mechanism. Pilchak et al. found that thousands of fatigue load cycles could contribute to the formation of a single facet, providing evidence to contradict the cleavage facet formation theory. ${ }^{[40]}$ Bridier et al. discuss observations of both basal and prismatic slip bands, and suggest fatigue cracks to have formed from these slip planes. ${ }^{[41]}$ In addition, Bantounas et al. found evidence suggesting facets on a high-cycle fatigue fracture surface to be associated with basal slip. ${ }^{\text {[42] }}$ Figure 4 demonstrated pole figures achieved from direct indexing of facets in the hydrogen-embrittled origin region. These pole figures suggested the plane of fracture to be a high index plane, such as (1017) or (1018), which are known hydrogen-habited planes. The facets in question contain structure, and are certainly not featureless, thus it is suggested that the formation of these low $\Delta \mathrm{K}$ features arise via a slip-induced mechanism, perhaps on one of the high index planes stated above.

Figure 11 demonstrates a representative overview of the general fractography observed at a crack length of $600 \mu \mathrm{m}$ (grain 4). Both secondary cracking, (a) and (b), and striated growth, (c) and (d), are plastic processes,
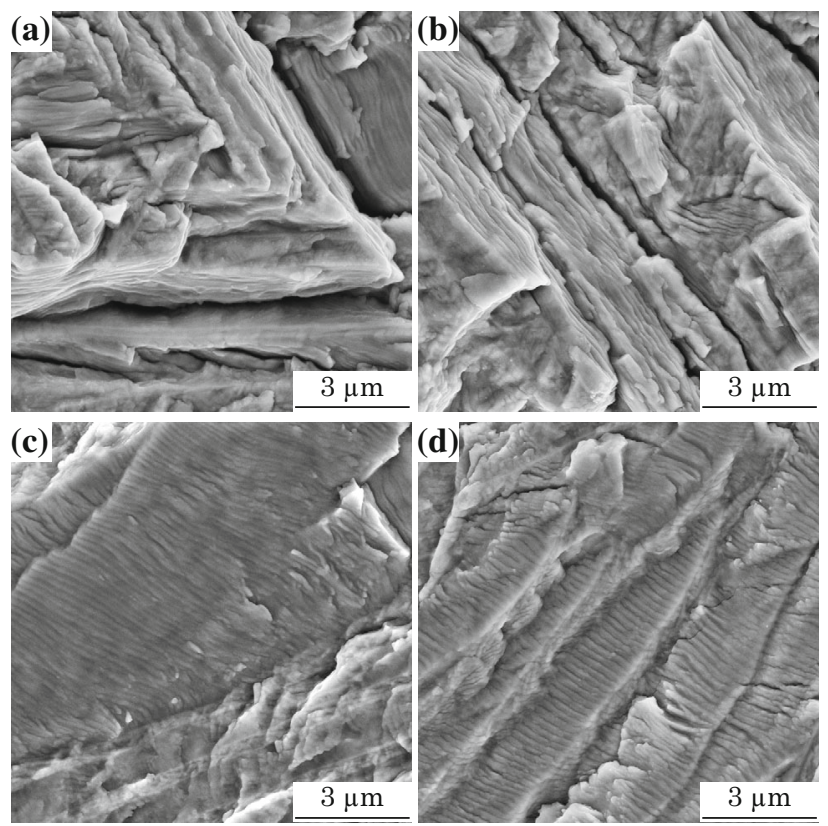

Fig. 11-Secondary electron images taken from the fatigue crack-propagation region, all at a crack length of $\sim 600 \mu \mathrm{m}$, close to the location from which the foil containing grain 4 was extracted: $(a)$ and (b) secondary cracking, (c) and (d) evidence of underlying microstructure as primary alpha lath structures and torn striations. indicating that the material at this crack length has undergone significant plasticity prior to crack propagation. Not only do the secondary cracks appear to be held open, but the fine ligaments of material between individual striations are observed to have torn apart, Figure 11(d), which is a further indication of significant plasticity. In contrast, the material within the hydrogen-embrittled region is largely facetted and has a macroscopically brittle appearance (grains 1 to 3); refer to Chapman et al., Figure 4. ${ }^{[2]}$

\section{Grain Orientation}

It has been well documented that under fatigue loading conditions, grains will deform via particular slip systems depending on their orientation. For single crystals, a specific slip system is favored depending on, 1 : the critical resolved shear stress (CRSS) necessary to initiate slip on the given system (also dependent on temperature), and 2: the Schmid factor, which is a function of grain orientation with respect to the loading axis. It is more complicated for polycrystals, where the orientation of neighboring grains, as well as the orientation of adjacent grain boundaries, can affect slip mechanisms. Bantounas et al. attempted to predict the active slip system of a given grain by introducing a term defined as a normalized Schmid factor, $m^{\prime}$, given by

$$
m^{\prime}=m \cdot\left(\tau_{\min } / \tau_{\langle u v t w\rangle\{h k i l\}}\right)
$$

where the $\left(\tau_{\min } / \tau_{\langle u v t w\rangle}\{h k i l\}\right)$ component is the ratio of the minimum CRSS to activate any slip system vs the CRSS of a particular slip system. ${ }^{[43}$ According to this rationale, it was expected that grains 1 and 2 would undergo $\langle a\rangle$ prismatic slip, and grain $3\langle a\rangle$ basal slip. Instead, we have suggested above that grains 1 and 2 underwent $\langle a\rangle$ basal slip and grain $3\langle a\rangle$ first-order pyramidal slip. It is suggested that this lack of conformity indicates a change in deformation mechanism, which may be attributed to solute hydrogen.

Since it is possible to disregard any effects relating to crack length, grains 1, 2, and 5 can be directly compared (hard orientation), with those for grains 3 and 4 (soft orientation); Table I. Although in each case, the grains are only slightly misorientated, their dislocation densities and distributions are very different. Considering their similar alignment to the loading axis, this disparity cannot be explained as a consequence of grain orientation. Instead, solute hydrogen at or near the crack tip during the fracture of grain 3 is suggested to affect the plastic behavior of the grain. This idea is reiterated, since grains 1 and 2 have a hard, rather than soft, orientation, but still demonstrate a similar dislocation distribution and density to grain 3 .

\section{Solute Hydrogen Diffusion}

This study has demonstrated a clear change in microdeformation mechanisms likely to be associated with solute hydrogen, and the consequent embrittlement via HELP. In this particular case, a naturally occurring hydrogen-embrittled fatigue crack origin has been 
investigated. Reactions leading to the production of hydrogen and its consequent adsorption are given in the accompanying paper, ${ }^{[29]}$ where it is explained there is a finite supply of hydrogen. It is also considered that only relatively low concentrations of hydrogen are involved in the mechanism, which may explain the lack of observed hydride phases. Bache et al. suggested that hydrogen may have the most significant effect on dislocation behavior when present in lower concentrations. ${ }^{[44]}$ Of course, hydrogen diffusivity is proportional to temperature. The rapid diffusion of hydrogen in the beta phase at elevated temperatures is the property that makes the HELP mechanism conceivable. For the bulk specimens discussed in this study, the test temperature was $450{ }^{\circ} \mathrm{C}$, which is sufficiently high to elevate hydrogen mobility. Another observation linked to test temperature was the inclined dislocation segments observed in grains 1 and 2. These segments were observed to have a different Burgers vector with respect to the majority of the linear dislocation array. When two edge dislocations intersect, one dislocation cuts through the glide plane of another, creating a jog. A jog will form in the glide plane of a mobile dislocation when its dislocation line vector is not parallel to the Burgers vector of the interacting dislocation. Hence, the jog will lie in the plane of the intersecting dislocation, and in this case, will have a different Burgers vector to other dislocations gliding in the original slip plane before intersection. It is suggested that the short inclined dislocation segments observed in grains 1 are 2 are formed as jogs. Motions of these jogs are a diffusion-controlled process only possible at elevated temperatures, such as those experienced here. Interestingly, the inclination of these jogs is $\sim 120 \mathrm{deg}$ to the basal trace, which is also the approximate angle between both the $\{1 \overline{1} 01\}$ and $\{\overline{1} \overline{1} 22\}$ pyramidal planes with the basal plane. Therefore, it is suggested that under high temperature, some edge dislocations moved out of their glide plane by climb on the pyramidal $\{1 \overline{1} 01\}\langle 11 \overline{2} 3\rangle$ or $\{\overline{1} \overline{1} 22\}\langle 11 \overline{2} 3\rangle$ slip systems.

\section{E. A Rationale for Lower Dislocation Density with Solute Hydrogen}

It is well documented that hydrogen increases the mobility of dislocations. ${ }^{[20,31-33,44]}$ Shih et al. explain that hydrogen enhances plasticity in the vicinity of a crack tip (HELP), altering the local deformation mechanism. Since localized microfracture could then occur at stresses below the macroscopic yield stress, it can be considered a locally brittle material response, which may explain the brittle faceted appearance at the hydrogen origin. ${ }^{[20]}$

The Orowan equation describes the relationship between strain rate $\dot{\epsilon}$, dislocation density $\rho_{m}$, average velocity $\bar{v}$ and magnitude of the Burgers vector $b$ of the mobile dislocations. ${ }^{[45]}$

$$
\dot{\epsilon}=b \rho_{\mathrm{m}} \bar{v}
$$

It has previously been discussed that grains 1 to 3 have a considerably lower dislocation density than grains 4 and
5. This has been attributed to solute hydrogen's presence ahead of the fatigue crack origin. This observation can be rationalized using the Orowan equation and the effect of hydrogen. If hydrogen enhances dislocation mobility, thereby raising the dislocation velocity, then at a given strain rate in the crack tip's plastic zone, this implies a reduction in the mobile dislocation density. Therefore, with the hydrogen enhancing the mobility of the dislocations, it follows that fewer dislocations should be observed. It is understood that hydrogen enters the lattice at elastic singularities, an example of which is the material immediately ahead of the advancing crack. As the hydrogen concentration is the highest at regions such as this, plasticity is constrained locally at the crack tip.

For grains 4 and 5, the opposite is observed. Without hydrogen in solution, dislocations are less mobile. Hence, for the same strain rate, many more dislocations will be observed, resulting in more dislocation interactions. The increased density meant that more dislocation interactions occurred, making jogs. As jogs effectively increase the length of the dislocation line, this hardens the material, as well as further impedes their motion; ${ }^{[46]}$ the more the dislocations cutting through the "forest" of dislocations, the harder the material becomes. This is commonly referred to as forest hardening, consistent with observations from grain 4 . It is generally considered that screw dislocations control deformation in the $\alpha$ phase, ${ }^{[30,47]}$ since edge segments are believed to move at least 100 times faster. ${ }^{[4]}$ It has also been suggested that solute hydrogen can "increase the propensity for edge character dislocations," $[33]$ explaining why the frequency of occurrence of edge dislocations may have been higher in the hydrogen-embrittled grains analyzed in this study. It also follows that since edge dislocations are considered to be highly mobile, ${ }^{[49]}$ their increased presence in the hydrogen-embrittled grains is consistent with the HELP mechanism and the Orowan equation.

\section{F. Consistency of the HELP Mechanism with the Observation of Macroscopic Hardening}

The HELP concept was first introduced by Beachem, ${ }^{[21]}$ and has since been the subject of extensive TEM investigations by Birnbaum, Shih, Sofronis, Robertson and coworkers. Shih et al., using in situ straining in the TEM, report that the velocity of dislocations near a crack tip in a thin foil is enhanced when hydrogen is added to an environmental cell under constant stress. ${ }^{[20]}$ Birnbaum and Robertson have since proposed that hydrogen shielding of dislocation-dislocation interactions would explain their observation of enhanced dislocation mobility. ${ }^{[31,33]}$ They believe that this elastic shielding allows dislocations to move at lower applied stress levels in the presence of hydrogen, resulting in localized flow softening at a crack tip.

In contrast, other studies ${ }^{[50-52]}$ have found that hydrogen in solution increases the macroscopic flow stress, implying solute hardening. The solubility of $\mathrm{H}$ in $\mathrm{Ti}$ at room temperature is approximately 0.1 at pet $(\sim 20$ ppmw). ${ }^{[53]}$ These studies suggest that this solute hardening is due to a dislocation pinning effect, although it 
should be noted that the hydrogen atom is small compared with the interstitial site, which is a drawback to this rationale. Beachem, observing hydrogen-assisted cracking and an associated reduction in fracture toughness, suggested that hydrogen unlocks dislocations, rather than pinning them. ${ }^{[21]}$

Therefore, it remains to rationalize how hydrogen, e.g., at a hot-salt stress corrosion crack tip, can reduce toughness, enhance dislocation mobility, and yet provide macroscopic hardening. A primary difference between a crack tip or thin foil and the bulk is the availability of a free surface. This could allow gliding dislocations to escape. Therefore, if appealing to the Orowan equation, the HELP mechanism gives rise higher glide velocities, and so to a lower dislocation density for a given strain rate, then in the bulk. this would result in larger glide lengths and more opportunity for dislocation tangling or forest hardening. However, in the presence of a free surface, this straining can occur without a gradual rise in dislocation density.

In addition, these observations are consistent with the reduction of $K_{\mathrm{Ic}}$ with hydrogen content-hydrogen embrittlement is a consequence of enhanced plasticity and dislocation motion at the crack tip, rather than requiring the alternative that decohesion (the formation of free surface) becomes easy even at the very low $\mathrm{H}$ contents in (solid) solution that pertain in the present case, as proposed by Lynch. ${ }^{[54]}$

Thus, the present observation of a lower dislocation density below a stress corrosion crack than that in low-cycle fatigue is consistent with both the observations of Shih and the HELP mechanism, and the observation of macroscopic flow hardening and reduced fracture toughness.

\section{G. Critical Diffusion Length}

The significant difference in dislocation density, and its association with solute hydrogen, has been discussed in detail. This was attributed to the ability of hydrogen to enhance dislocation motion. It is also observed, that for hydrogen-embrittled grains, dislocations appear to be restricted to within a 3- $\mu \mathrm{m}$ depth beneath the fracture surface. However, for grain 4 (crack propagation), the high density of dislocations continue throughout the length of the primary alpha lath, which intersects the fracture surface at $\sim 90 \mathrm{deg}$. This observation can be explained by considering the competitive processes of hydrogen charging and hydrogen diffusion. The reader is referred to the accompanying paper by Chapman et al. ${ }^{[29]}$ for a detailed explanation of these reactions, but the important points are summarized here.

When the rate of hydrogen charging exceeds the rate of hydrogen diffusion, the local hydrogen concentration present immediately in front of the crack is sufficiently high to embrittle the material locally, via the HELP mechanism. The grain experiences highly localized enhanced plastic strain and undergoes deformation in the vicinity of the crack tip, leading to crack advance.

It is proposed that the localized nature of the deformation causes HELP to act over only the portion of the grain closest to the newly created fracture surface.
It follows that the lower region of the primary alpha lath will not experience enhanced plasticity before the crack front advances. Therefore, assuming constant hydrogen charging and diffusion rates at a given temperature, it is postulated that there is a critical diffusion distance, beyond which charging is insufficient to cause embrittlement. Hence, because HELP is not active beyond this critical diffusion length, $L_{\mathrm{D}}$, hydrogen-enhanced fracture mechanisms are constrained to the uppermost material, estimated at $3 \mu \mathrm{m}$ for grains 1 to 3 . It should also be noted that, when $L_{\mathrm{D}}$ is of the same order of magnitude as the crack growth increment per fatigue cycle, there is no possibility for hydrogen accumulation over multiple cycles. Recent work by Gaddam et al., discusses this area in more detail. ${ }^{[55]}$

Alternatively, turning to the AIDE hypothesis, we first consider that at $450{ }^{\circ} \mathrm{C}$, the hydrogen diffusion rate and solubility are both likely high enough for diffusion to a 3- $\mu \mathrm{m}$ depth to occur, in contrast to the AIDE situation pertaining to ambient temperatures and high crack growth rates considered by Lynch. ${ }^{[23,54]}$ As previously noted, there is also the possibility of the $\beta$ phase acting as a fast diffusion path. ${ }^{[28]}$ However, as has been more recently noted by Lynch, ${ }^{[56]}$ it remains possible that dislocation nucleation from hydrogen at the surface might then result in propagation into the depth of the material, in a HELP-like fashion. However, in our view, the change in dislocation morphology (particularly those traveling in ribbons in Figure. 7) suggests that something more is occurring rather than simply hydrogen-stimulated dislocation emission.

\section{CONCLUSIONS}

The dislocation mechanisms underlying a naturally initiated hot-salt stress corrosion crack have been elucidated and compared with those observed in conventional low-cycle fatigue. The following conclusions can be drawn:

1. Hydrogen-embrittled grains do not appear to conform to the predictions made by the normalized Schmid factor method, as proposed by Bantounas et al. ${ }^{[43]}$

2. It is proposed that hydrogen in the vicinity of the crack tip modifies dislocation behavior (which could be through the AIDE or HELP mechanism).

3. Irrespective of the grain orientation, the dislocation density is consistently, and significantly, lower in hydrogen-embrittled grains compared to those from the fatigue crack-propagation region and LCF origin.

4. The effect of crack length is excluded as a contributing factor to the increased dislocation density observed in the LCF propagation region.

5. The Orowan equation is used to explain the reduction in dislocation density seen within hydrogen-embrittled grains, by considering the elevated dislocation mobility attributed to the onset of HELP at the crack tip.

6. A rationale for the observed flow softening in thin foils $v s$ that of macroscopic hardening in bulk specimens is proposed. 
7. A term defined as the critical diffusion length is introduced. It is suggested that the competitive reaction between hydrogen charging and hydrogen diffusion determines the maximum depth beneath the fracture surface that is affected by solute hydrogen (with the HELP mechanism in view).

\section{ACKNOWLEDGMENTS}

The authors wish to acknowledge support from Rolls-Royce plc throughout this work. We would also like to thank the numerous people involved, who are based at Rolls-Royce Derby, Bristol, and Dahlewitz. The authors would also like to acknowledge the very useful discussions with Professor Ian Jones. Finally, we are grateful for the funding from the EPSRC; Grants EP/H004882/1 and EP/K034332/1, as well as a DTA CASE conversion EP/J500239/1 for T.P.C.

\section{REFERENCES}

1. R. Boyer: Adv. Perform. Mater., 1995, vol. 2, pp. 349-68.

2. R. Boyer: Mater. Sci. Eng. A, 1996, vol. A213, pp. 103-14.

3. J. Williams and E.J. Starke: Acta Mater., 2003, vol. 51, pp. 5775-99.

4. G. Crook and M. Horlor, eds.: Rolls-Royce-The Jet Engine, 1st ed., Rolls-Royce plc, Derby, UK, 1996.

5. G. Lütjering and J. Williams: Titanium, Springer Science, Berlin, 2003.

6. M. Blackburn and J. Williams: Proceedings of Conference on Fundamental Aspects of Stress Corrosion Cracking, 1969.

7. R. Ondrejcin: Chlorine gas and fluoride ion in hot-salt stress corrosion cracking of titanium-aluminium alloys, NASA DP-1179, 1969.

8. V. Petersen and H. Bomberger: Stress Corros. Crack. Titan. ASTM Spec. Tech. Publ., 1966, vol. 397, pp. 80-94.

9. S. Rideout, M. Louthan, Jr., and C. Selby: Stress Corros. Crack. Titan. ASTM Spec. Tech. Publ., 1966, vol. 397, pp. 137-51.

10. H. Logan, M. McBee, C. Bechtoldt, B. Sanderson, and G. Ugiansky: Stress Corros. Crack. Titan. ASTM Spec. Tech. Publ., 1966, vol. 397, pp. 215-29.

11. J. Myers and J. Hall: Corros. NACE, 1977, vol. 33, pp. 252-57.

12. M. Garfinkle: Metal. Trans., 1973, vol. 4, pp. 1677-86.

13. M. Pustode, V. Raja, and N. Paulose: Corros. Sci., 2014, vol. 82, pp. 181-96.

14. A. Pilchak, A. Young, and J. Williams: Corros. Sci., 2010, vol. 52 , pp. 3287-96.

15. B.F. Brown: Stress Corrosion Cracking in High Strength Steels and in Titanium and aluminium Alloys, Naval Research Laboratory, 1972.

16. E. Tal-Gutelmacher and D. Eliezer: Mater. Trans., 2004, vol. 45, pp. 1594-600.

17. T. MacKay: Metal. Trans., 1971, vol. 2, pp. 2299-302.

18. H. Nelson: Aqueous Chloride Stress Corrosion Cracking of Titanium-A Comparison with Environmental Hydrogen Embrittlement, NASA TM X-62314, 1973.

19. H. Nelson: Metal. Trans. A, 1976, vol. 7A, pp. 621-27.

20. D. Shih, I. Robertson, and H. Birnbaum: Acta Metal., 1988, vol. 36, pp. 111-24.
21. C. Beachem: Metal. Trans., 1972, vol. 3, pp. 437-51.

22. I.M. Robertson, P. Sofronis, A. Nagao, M.L. Martin, S. Wang, D.W. Gross, and K.E. Nygren: Metal. Mater. Trans. B, 2015, vol. 46B, pp. 1085-03.

23. S.P. Lynch: Corros. Rev., 2012, vol. 30, pp. 105-23.

24. SP Lynch: Metal. Mater. Trans. A, 2013, vol. 44A, pp. 1209-29.

25. S.P. Lynch: Acta Metal., 1984, vol. 32, pp. 79-90.

26. E. Tal-Gutelmacher and D. Eliezer: J. Mater. Overv., 2005, pp. 46-49.

27. H. Nelson, D. Williams, and J. Stein: Metal. Trans., 1972, vol. 3, pp. $469-75$.

28. P. Pao and J. O’Neal: J. Nucl. Mater., 1984, vol. 122 (123), pp. 1587-91.

29. T. Chapman, R. Chater, E. Saunder, A. Walker, T. Lindley, and D. Dye: Corr. Sci., 2015, vol. 96, pp. 87-101.

30. F. Mignot, V. Doquet, and C. Sarrazin-Baudoux: Mater. Sci. Eng. A, 2004, vol. 380, pp. 308-19.

31. P. Birnbaum and H.K. Sofronis: Mater. Sci. Eng. A, 1994, vol. 176, pp. 191-202.

32. P. Ferreira, I. Robertson, and H. Birnbaum: Acta Mater., 1998, vol. 46, pp. 1749-57.

33. I. Robertson: Eng. Fract. Mech., 2001, vol. 65, pp. 649-73.

34. V. Sinha, M. Mills, and J. Williams: Metal. Mater. Trans. A, 2006, vol. 37A, pp. 2015-26.

35. V. Sinha, M. Mills, and J. Williams: J. Mater. Sci., 2007, vol. 42, pp. 8334-41.

36. G. Themelis, S. Chikwembani, and J. Weertman: Mater. Charact., 1990, vol. 24, pp. 27-40.

37. L. Giannuzzi and F. Stevie: Micron, 1999, vol. 30, pp. 197-204.

38. D. Kiener, C. Motz, M. Rester, M. Jenko, and G. Dehm: Mater. Sci. Eng. A, 2007, vol. 459, pp. 262-72.

39. N. Paton and R. Spurling: Metal. Trans. A, 1976, vol. 7, pp. 1769-74.

40. A. Pilchak, A. Bhattacharjee, A. Rosenberger, and J. WIlliams: Int. J. Fatigue, 2009, vol. 31, pp. 989-94.

41. F. Bridier, P. Villechaise, and J. Mendez: Acta Mater., 2008, vol. 56 , pp. 3951-62.

42. I. Bantounas, D. Dye, and T. Lindley: Acta Mater., 2010, vol. 58, pp. 3908-18.

43. I. Bantounas, D. Dye, and T. Lindley: Acta Mater., 2009, vol. 57, pp. 3584-95.

44. M. Bache, W. Evans, and H. Davies: J. Mater. Sci., 1997, vol. 32, pp. 3435-42.

45. D. Hull and D. Bacon: Introduction to Dislocations, Butterworth-Heinemann, London, 2001.

46. M. Sangid: Int. J. Fatigue, 2013, vol. 57, pp. 58-72.

47. P. Castany, F. Pettinari-Sturmel, J. Douin, and A. Coujou: Mater. Sci. Eng. A, 2008, vols. 483-484, pp. 719-22.

48. P. Castany, F. Pettinari-Sturmel, J. Crestou, J. Douin, and A. Coujou: Acta Mater., 2007, vol. 55, pp. 6284-91.

49. S. Naka, A. Lasalmonie, P. Costa, and L. Kubin: Philos. Mag. A, 1988, vol. 57, pp. 717-40.

50. H. Matsui, H. Kimura, and S. Moriya: Mater. Sci. Eng., 1979, vol. 40, pp. 207-16.

51. W. Xie, X. Liu, W. Chen, and H. Zhang: Comput. Mater. Sci., 2011, vol. 50, pp. 3397-02.

52. F. Beck: Effect of hydrogen on the mechanical properties of titanium and its alloys, Technical Report CR-134796, NASA, 1975.

53. H. Conrad: Prog. Mater. Sci., 1981, vol. 26, pp. 123-403.

54. S. Lynch: Corrosion-NACE, Nashville, TN, 2007.

55. R. Gaddam, M. Perderson, R. Hörnqvist, and M.-L. Antti: Corros. Sci., 2014, vol. 78, pp. 378-83.

56. S.P. Lynch: in Gaseous Hydrogen Embrittlement of Metals in Energy Technologies-Volume 1: The Problem, Its Characterisation and Rffects on Particular Alloy Classes, R.P. Gangloff and B.P. Somerday, eds., Cambridge, 2012, pp. 274-346. 